\title{
DOPPLER-FREE MULTIPHOTON ABSORPTION IN ICOSAHEDRAL MOLECULES
}

\author{
David L. ANDREWS and Kevin P. HOPKINS \\ School of Chemical Sciences, University of East Anglia, Norwich NR4 7TJ, UK
}

Received 29 January 1988; in final form 15 February 1988

\begin{abstract}
It is shown that with counter-propagating laser beams of identical circular polarisation, symmetry-preserving transitions in icosahedral molecules produce totally Doppler-free lines in two-photon and four-photon absorption spectra. In the four-photon case, this feature cannot occur for molecules of any other symmetry, and therefore characterises an icosahedral species.
\end{abstract}

\section{Introduction}

Until recently the chemical physics of icosahedral species was a matter of largely academic interest, since only the borohydride anion $\mathrm{B}_{12} \mathrm{H}_{12}^{2-}$ was known to have this structure. However, with the synthesis [1] of dodecahedrane, $\mathrm{C}_{20} \mathrm{H}_{20}$, and the characterisation [2] of Buckminsterfullerene, $\mathrm{C}_{60}$, there is now a new significance to the study of icosahedral compounds in the gas phase. In the more widely studied case of $\mathrm{C}_{60}$, there are two possible structures [3], but icosahedral symmetry has been positively confirmed by the study of various spectroscopic properties (see for example refs. [4-7]), and also by recent X-ray cyrstallographic measurements $[8]$. Alongside the experimental work, there has been renewed theoretical interest in the electronic, vibrational, and rotational states of such compounds [9-23].

It is the purpose of this paper to draw attention to a novel feature predicted by theory in the multiphoton spectroscopy of such species. Doppler-free multiphoton absorption spectroscopy is based on a firstorder cancellation of Doppler frequency shifts. This is accomplished by arranging that the wave-vectors of the absorbed photons give a vector sum of zero [24]. This paper is concerned with two simple cases; (i) the two-photon absorption of counter-propagating photons of equal frequency, and (ii) the fourphoton absorption of two pairs of counter-propagating photons. In both cases it is shown that the use of circularly polarised light produces a feature which characterises icosahedral symmetry.

\section{Two-photon absorption}

We begin by reviewing the irreducible tensor calculus for the simpler case of two-photon absorption. The rate of absorption, obtained by means of the standard methods of quantum electrodynamics [25], can be expressed in the form

$\Gamma=K_{2}\left|e_{1 i} e_{2 j} T_{i j}\right|^{2}$.

Here $e_{1}$ and $e_{2}$ are the polarisation vectors of the two absorbed photons, $\mathbf{T}$ is the two-photon molecular response tensor for the transition, and there is implied summation over the repeated indices $i, j=(x, y, z)$. The symmetry analysis of the above expression is facilitated by recasting both the "polarisation tensor" $\left(e_{1 i} e_{2 j}\right)$ and the molecular tensor in irreducible form.

The decomposition of a general, reducible, second-rank Cartesian tensor $S_{i j}$, into irreducible parts takes the form [25]

$S_{i j}=S_{i j}^{(0)}+S_{i j}^{(1)}+S_{i j}^{2)}$.

Here, $S_{i j}^{(0)}$ is a weight-0 tensor which transforms under the operations of the full rotation group as a sca$\operatorname{lar} ; S_{i j}^{(1)}$ is a weight-1 tensor, which transforms as a pseudo-vector (i.e. its components have the same transformation properties as the rotations, $R_{x}, R_{y}$, $R_{z}$ ); and $S_{i j}^{(2)}$ is a weight-2 tensor which transforms as a second-rank, symmetric, traceless tensor (i.e. its components transform in the same way as $x y, x z, y z$, $x^{2}-y^{2}$ and $\left.2 z^{2}-x^{2}-y^{2}\right)$.

Since index-symmetry properties dictate that the inner product of two irreducible tensors of the same 
rank but different weight is zero, the cross-terms in eq. (1) vanish and we have

$$
\begin{aligned}
\Gamma & =K_{2} \mid\left(e_{1 j} e_{2 j}\right)^{(0)} T_{i j}^{(0)} \\
& +\left(e_{1 i} e_{2 j}\right)^{(1)} T_{i j}^{(1)}+\left.\left(e_{1 i} e_{2 j}\right)^{(2)} T_{i j}^{(2)}\right|^{2} .
\end{aligned}
$$

If a particular molecular transition is to be observed, it is necessary that one or more of the irreducible molecular tensors transforms under the same irreducible representation as the transition. In particular, a symmetry-preserving two-photon transition in a cubic or icosahedral species (in which the product of the initial and final states is spanned by the $A_{1 g}$ irreducible representation in $\mathrm{O}_{\mathrm{h}}$, or the $\mathrm{A}_{\mathrm{g}}$ irreducible representation in $I_{h}$ ), will only be observed in the spectrum if the corresponding weight 0 contributions to the rate equation are non-zero. (In molecules of any other symmetry, weight- 2 contributions also transform under the totally symmetric representation, and therefore the absence of a weight -0 term does not necessarily imply that the transition is forbidden.)

The explicit form of each irreducible tensor contribution is obtained through use of the relations

$$
\begin{aligned}
& T_{i j}^{(0)}=\frac{1}{3} \delta_{i j} T_{k k}, \\
& T_{i j}^{(1)}=\frac{1}{2}\left(T_{i j}-T_{j i}\right), \\
& T_{i j}^{(2)}=\frac{1}{2}\left(T_{i j}+T_{j i}\right)-\frac{1}{3} \delta_{i j} T_{k k}, \\
& \left(e_{1 i} e_{2 j}\right)^{(0)}=\frac{1}{3} \delta_{i j}\left(e_{1} \cdot e_{2}\right), \\
& \left(e_{1 i} e_{2 j}\right)^{(1)}=\frac{1}{2}\left[\left(e_{1 i} e_{2 j}\right)-\left(e_{1 j} e_{2 i}\right)\right], \\
& \left(e_{1 i} e_{2 j}\right)^{(2)}=\frac{1}{2}\left[\left(e_{1 i} e_{2 j}\right)+\left(e_{1 j} e_{2 i}\right)\right]-\frac{1}{3} \delta_{i j}\left(e_{1} \cdot e_{2}\right) .
\end{aligned}
$$

Since the molecular tensor is index-symmetric if the two absorbed photons are of the same frequency, $T_{i j}^{(1)}$ vanishes and there is no weight-1 contribution to the process. Clearly, the presence or absence of the weight -0 component depends on the value of the dot product $\left(e_{1} \cdot e_{2}\right)$.

With counter-propagating plane polarised beams the value of the dot product $\left(e_{1} \cdot e_{2}\right)$ is determined by the angle between the two polarisation vectors. In the particular case where both photons are derived from a single beam, the dot product must always have the value of unity. Hence the rate equation for a symmetry-preserving transition will always contain a weight -0 contribution arising from the absorption of two photons from the same beam. Thus whilst the absorption of two counter-propagating photons will indeed produce a Doppler-free signal, the overall absorption profile will be one in which this Dopplerfree line is superimposed on a band which carries the usual Doppler broadening. Special features arise with circularly polarised radiation, however, and we therefore concentrate on this case below.

When two counter-propagating circularly polarised laser beams are employed, $\left(e_{1} \cdot e_{2}\right)$ is zero if both photons are derived from the same beam, since $e^{(\mathrm{L} / \mathrm{R})}=(1 / \sqrt{2})(\hat{\imath} \pm \mathrm{i} \hat{\jmath})$. The absorption of two copropagating photons therefore cannot induce a symmetry-preserving transition in $\mathrm{O}_{h}$ or $\mathrm{I}_{h}$ molecules: hence there is no Doppler-broadened background for such transitions (see table 1). A physical explanation for this behaviour lies in the fact that any two co-propagating circularly polarised photons carry a net spin angular momentum of $2 \hbar$, and thus can only be absorbed in two-photon transitions which result in the transfer of this angular momentum to the absorber. Symmetry-preserving transitions (i.e. transitions of weight- 0 character) in molecules of cubic or icosahedral symmetry can, however, only occur if there is no net uptake of angular momentum, and thus can only be induced by the absorption of counterpropagating photons. For the case where the beams are circularly polarised with opposite helicity, there is also no weight 0 contribution to $\left(e_{1} \cdot e_{2}\right)$, from counter-propagating photons since a left-circularly polarised photon moving from left to right has the same polarisation vector as a right-circularly polarised photon moving from right to left, and vice versa. Hence symmetry-preserving transitions are in this case entirely forbidden.

However with counter-propagating beams of the same handedness, a weight- 0 contribution from $\left(e_{1} \cdot e_{2}\right)$ is observed when one photon is derived from each beam; thus whilst the absorption of two photons from either beam, i.e. co-propagating photons, is a forbidden process, the absorption of counterpropagating photons is allowed. Hence under this particular experimental arrangement, a Doppler-free absorption band occurs in the two-photon spectrum without the normal Doppler-broadened background. This is the only experimental configuration whereby a totally symmetric transition in these point groups can produce a Doppler-free peak in the two-photon absorption spectrum: the effect thus characterises 
Table 1

Form of $S_{i j}^{(0)}$ and $\left(e_{1} \cdot e_{2}\right)$ for all possible cases of two-photon absorption with counter-propagating circularly polarised beams: $e_{1}$ and $e_{2}$ denote the polarisation vectors of the two absorbed photons, whilst $e$ and $e^{\prime}$ denote the polarisations of the two beams. A weight-0 transition is only allowed when $\left(e_{1} \cdot e_{2}\right) \neq 0$

$$
\text { Form of } s_{i j}^{(0)} \quad\left(e_{1} \cdot e_{2}\right)
$$

$\begin{array}{cll}\text { counter-propagating beams of the same helicity } & & \\ \text { two photons absorbed from the same beam } & \left(e_{1 i} e_{2 j}\right)^{(0)} & 0 \\ & \left(e_{1 i}^{\prime} e_{2 j}^{\prime}\right)^{(0)} & 0 \\ \text { one photon absorbed from each beam } & \left(e_{1 i} e_{2 j}\right)^{(0)} & 1 \\ \text { counter-propagating beams of opposite helicity } & & \\ \text { two photons absorbed from the same beam } & \left(e_{1 i} e_{2 j}\right)^{(0)} & 0 \\ & \left(e_{1 i}^{\prime} e_{2 j}^{\prime}\right)^{(0)} & 0 \\ & \left(e_{1 i} e_{2 j}^{j}\right)^{(0)} & 0\end{array}$

either cubic or icosahedral symmetry. A similar effect is a well-known feature of Doppler-free atomic spectroscopy [24].

\section{Four-photon absorption}

Whilst there is no analogue to this behaviour in three-photon absorption, similar remarks can be made concerning the four-photon case. The rate of four-photon absorption can be expressed as

$$
\Gamma=K_{4}\left|e_{1 i} e_{2 j} e_{3 k} e_{4 l} T_{i j k l}\right|^{2},
$$

where $e_{1}, e_{2}, e_{3}$ and $e_{4}$ are the polarisation vectors of the four absorbed photons, and $T_{i j k l}$ is the four-photon molecular response tensor for the transition. The wcight -0 contribution to the overall rate then arises from

$\Gamma^{(0)}=K_{4}\left|\left(e_{1 i} e_{2 j} e_{3 k} e_{4 l}\right)^{(0)} T(0)\right|^{2}$,

where the explicit forms of the irreducible tensors are given by [26]

$T_{i j k l}^{\{0\}}=\left[\begin{array}{l}\delta_{i j} \delta_{k l} \\ \delta_{i k} \delta_{j l} \\ \delta_{i l} \delta_{j k}\end{array}\right]^{\mathrm{T}}\left[\begin{array}{rrr}4 & -1 & -1 \\ -1 & 4 & -1 \\ -1 & -1 & 4\end{array}\right]\left[\begin{array}{l}T_{m m n n} \\ T_{m n m n} \\ T_{m n n m}\end{array}\right]$

and

$$
\begin{aligned}
& \left(e_{1 i} e_{2 j} e_{3 k} e_{4 l}\right)^{(0)} \\
& \quad=\left[\begin{array}{c}
\delta_{i j} \delta_{k l} \\
\delta_{i k} \delta_{j l} \\
\delta_{i l} \delta_{j k}
\end{array}\right]^{\mathrm{T}}\left[\begin{array}{rrr}
4 & -1 & -1 \\
-1 & 4 & -1 \\
-1 & -1 & 4
\end{array}\right]\left[\begin{array}{l}
\left(e_{1} \cdot e_{2}\right)\left(e_{3} \cdot e_{4}\right) \\
\left(e_{1} \cdot e_{3}\right)\left(e_{2} \cdot e_{4}\right) \\
\left(e_{1} \cdot e_{4}\right)\left(e_{2} \cdot e_{3}\right)
\end{array}\right]
\end{aligned}
$$

The presence, or absence, of a weight- 0 contribution to the spectrum thus depends on a non-zero value for at least one of the terms in the right-hand bracket of eq. (13). The values of these terms for a number of combinations of beam polarisations are presented in table 2. As in the case of two-photon absorption, employing counter-propagating beams of opposite helicity produces no weight- 0 contribution. With two counter-propagating circularly polarised beams of the same handedness, the only allowed mechanism for a four-photon symmetry-preserving transition involves absorbing a pair of photons from each beam. Once again this results in each such transition producing a Doppler-free line in the spectrum. However, in this case the effect is limited to icosahedral species: in molecules of any other symmetry, there are no cases where a weight -0 contribution arises without other weights being simultaneously represented [27].

Our results show that in an experiment based on counter-propagating laser beams of identical frequency and circular polarisations, an entirely Doppler-free line in a four-photon absorption spectrum should unambiguously signify icosahedral symmetry. A note of caution needs to be sounded on two points, however. First, we note that in a particular molecule certain four-photon transitions may involve additional tensor contributions of weight 2 or 4 which, although allowed, may be weak compared to the weight -0 contribution. This could produce a false positive result with the appearance of an essentially Doppler-free line which would normally characterise a pure weight- 0 transition. This problem might be overcome by confirming that the line 
Table 2

Form of $S_{i j k}^{(0)}$ and the three quantities $A=\left(e_{1} \cdot e_{2}\right)\left(e_{3} \cdot e_{4}\right), B=\left(e_{1} \cdot e_{3}\right)\left(e_{2} \cdot e_{4}\right)$ and $C=\left(e_{1} \cdot e_{4}\right)\left(e_{2} \cdot e_{3}\right)$, for all possible cases of four-photon absorption with counter-propagating circularly polarised beams: $e_{1}, e_{2}, e_{3}$ and $e_{4}$ denote the polarisation vectors of the four absorbed photons, whilst $e$ and $e^{\prime}$ denote the polarisations of the two beams. A weight- 0 transition is only allowed if $A, B$ or $C \neq 0$

\begin{tabular}{|c|c|c|c|c|}
\hline & Form of $S(0)$ & $A$ & $B$ & $C$ \\
\hline \multicolumn{5}{|l|}{ counter-propagating beams of the same helicity } \\
\hline \multirow[t]{2}{*}{ four photons absorbed from one beam } & $\left(e_{1 i} e_{2 j} e_{3 k} e_{4 l}\right)^{(0)}$ & 0 & 0 & 0 \\
\hline & $\left(e_{1 i}^{\prime} e_{2 j}^{\prime} e_{3 k}^{\prime} e_{4 l}^{\prime}\right)^{(0)}$ & 0 & 0 & 0 \\
\hline \multirow[t]{2}{*}{ three photons absorbed from one beam } & $\left(e_{1 i} e_{2 j} e_{3 k} e_{4 l}^{\prime}\right)^{(0)}$ & 0 & 0 & 0 \\
\hline & $\left(e_{1 i}^{\prime} e_{2 j}^{\prime} e_{3 k}^{\prime} e_{4 l}\right)^{(0)}$ & 0 & 0 & 0 \\
\hline two photons absorbed from each beam & $\left(e_{1 i} e_{2 j} e_{3 k}^{\prime} e_{4 l}^{\prime}\right)^{(0)}$ & 0 & 1 & 1 \\
\hline \multicolumn{5}{|l|}{ counter-propagating beams of opposite helicity } \\
\hline any combination & any & 0 & 0 & 0 \\
\hline
\end{tabular}

disappears when a single circularly polarised laser beam is used for the excitation (since, as table 2 shows, the weight- 0 term for a symmetry-preserving transition then disappears). Secondly, the need to distinguish Doppler-free lines with and without Doppler-broadened background might prove experimentally difficult. However, we are confident that the considerable ingenuity of the spectroscopists involved in this field can be successfully brought to bear on the problem.

\section{Acknowledgement}

We thank Professor Richard Dixon for helpful remarks on the experimental aspects of our work. KPH also gratefully acknowledges financial support from the Science and Engineering Research Council.

\section{References}

[1] L.A. Paquette, R.J. Ternansky, D.W. Balough and G. Kentagen, J. Am. Chem. Soc. 105 (1983) 5446.

[2] H.W. Kroto, J.R. Heath, S.C. O'Brien, R.F. Curl and R.E. Smalley, Nature 318 (1985) 162.

[3] T.I. Shibuya and M. Yoshitani, Chem. Phys. Letters 137 (1987) 13.

[4] E.A. Rohlfing, D.M. Cox and A. Kaldor, J. Chem. Phys. 81 (1984) 3322.

[5] S.H. Yang, C.L. Pettiette, J. Conceicao, O. Cheshnovsky and R.E. Smalley, Chem. Phys. Letters 139 (1987) 233.

[6] J.R. Heath, R.F. Curl and R.E. Smalley, J. Chem. Phys. 87 (1987) 4236.
[7] I. László and L. Udvardi, Chem. Phys. Letters 136 (1987) 418.

[8] S. Iijima, J. Phys. Chem. 91 (1987) 3466.

[9] R.A. Davidson, Theoret. Chim. Acta 58 (1981) 193.

[10] W.G. Harter and D.E. Weeks, Chem. Phys. Letters 132 (1986) 387.

[11] D.S. Marynick and S. Estreicher, Chem. Phys. Letters 132 (1986) 383.

[12] D.J. Klcin, T.G. Schmalz, G.E. Hitc and W.A. Seitz, J. Am. Chem. Soc. 108 (1986) 1301.

[13] R.C. Haddon, L.E. Brus and K. Raghavachari, Chem. Phys. Letters 125 (1986) 459.

[14] M.D. Newton and R.E. Stanton, J. Am. Chem. Soc. 108 (1986) 2469.

[15] A.D.J. Haymet, J. Am. Chem. Soc. 108 (1986) 319.

[16] R.L. Disch and J.M. Schulman, Chem. Phys. Letters 125 (1986) 465.

[17] K.E. Schriver, A.J, Paguia, M.Y. Hahn, E.C. Honea, A.M. Camarena and R.L. Whetten, J. Phys. Chem. 91 (1987) 3131 .

[18] B.W. van de Waal, J. Chem. Phys. 86 (1987) 5660.

[19] S. Larsson, A. Volosov and A. Rosén, Chem. Phys. Letters 137 (1987) 501.

[20] H.P. Lüthi and J. Almlöf, Chem. Phys. Letters 135 (1987) 357.

[21] J.R. Heath, S.C. O'Brien, R.F. Curl, H.W. Kroto and R.E. Smalley, Comments Condensed Matter Phys. 13 (1987) 119.

[22] Z.C. Wu, D.A. Jelski and T.F. George, Chem. Phys. Letters 137 (1987) 291.

[23] S.J. Cyvin, E. Brendsdal, B.N. Cyvin and J. Brunvoll, Chem. Phys. Letters 143 (1988) 377.

[24] M.D. Levenson, Introduction to nonlinear spectroscopy (Academic Press, New York, 1982).

[25] D.P. Craig and T. Thirunamachandran, Molecular quantum electrodynamics (Academic Press, New York, 1984).

[26] D.L. Andrews and W.A. Ghoul, Phys. Rev. A 25 (1982) 2647.

[27] D.L. Andrews and W.A. Ghoul, J. Chem. Phys. 75 (1981) 530. 